\section{Congratulatory Comment}

Check for updates

\title{
Congratulatory comment from Association of Pacific Rim Universities Global Health Program
}

\author{
Mellissa Withers
}

Director, Association of Pacific Rim Universities (APRU) Global Health Program

(c) 2019 Korean Society of Global Health. This is an Open Access article distributed under the terms of the Creative Commons Attribution Non-Commercial License (https:// creativecommons.org/licenses/by-nc/4.0/) which permits unrestricted non-commercial use, distribution, and reproduction in any medium, provided the original work is properly cited.
As the world and its economies become globalized, our global community becomes smaller and the health challenges we now face transcend geographical boundaries and cultures. Global health has had many laudable achievements. Unparalleled improvements in living conditions, poverty reduction, and life expectancies were made in the last century. More recently, we have seen major reductions in extreme poverty and maternal and infant mortality, incredible innovations in vaccines and technology, and improved access to lifesaving drugs. However, many more challenges ahead. Global phenomena such as aging, urbanization, migration, environmental degradation, rising rates of chronic diseases, and social and economic inequalities, undermine health and threaten our survival. Solving these complex problems will require immense collaboration to safeguard health. To be a global health professional in these times is exciting and daunting. We are in the position to transform the health of our global population. In fact, I believe we have a moral imperative to use our resources, skills, and expertise to do so.

Confronting the health challenges of our modern world requires challenging conventional wisdom with new ideas that reflect the changing global landscape. The launch of the Journal of Global Health Science (JGHS) is an important step in advancing global health by creating a platform to share and debate scholarly research from around the world. The dissemination of research is critical to building an evidence-base, sharing lessons learned, and maximizing efficiency and costs by avoiding duplicative efforts. I am pleased to see that the focus of the JGHS is under-served populations in low- and middle-income economies and marginalized groups in high-income economies. The JGHS will expand the opportunity to share high quality scientific work through an open-access format.

I congratulate Dr. Juhwan Oh, Editor-in-Chief, for this impressive accomplishment. Dr. Oh has been a long-standing member of the advisory group of the Global Health Program of the Association of Pacific Rim Universities (APRU). As the only network of leading universities linking the Americas, Asia and Australasia, APRU is the voice of knowledge and innovation for the Asia-Pacific region. We bring together thought leaders, researchers, and policymakers to exchange ideas and collaborate on effective solutions to the challenges of the 21st century. As the Director of APRU Global Health Program, I have witnessed Dr. Oh's vision, leadership, and dedication to advancing the field of global health. I am proud to serve on the editorial board of the JGHS with many other scholars from around the world and look forward to witnessing the growth and success of the JGHS. 